Cite this: Soft Matter, 2014, 10, 4324

\title{
The impact range for smooth wall-liquid interactions in nanoconfined liquids
}

\author{
Trond S. Ingebrigtsen* and Jeppe C. Dyre
}

Bulk and nanoconfined liquids have very different physics; for instance, nanoconfined liquids show stratification and position-dependent relaxation processes. A number of similarities between bulk and nanoconfined liquids have nevertheless been reported in computer simulations during the last decade. Inspired by these observations, we present results from molecular dynamics computer simulations of four nanoconfined liquids (the single-component Lennard-Jones liquid, the Kob-Andersen binary Lennard-Jones mixture, an asymmetric dumbbell model, and the Dzugutov liquid) demonstrating also a microscopic similarity between bulk and nanoconfined liquids. The results show that the interaction range for the wall-liquid and liquid-liquid interactions of the nanoconfined liquid is identical to that of the bulk liquid if the liquid is "Roskilde simple" in bulk as well as nanoconfinement, i.e., exhibits strong correlations between virial and potential-energy equilibrium fluctuations in the NVT ensemble. Under this condition, interactions beyond the first coordination shell may be ignored, in particular for the wallliquid interactions. This is shown not to be the case for non-Roskilde-simple liquids.

Received 16th September 2013 Accepted 27th February 2014

DOI: $10.1039 / \mathrm{c} 3 \mathrm{sm} 52441 \mathrm{~h}$

www.rsc.org/softmatter study simple inhomogenous fluids build upon free-energy expressions for the bulk fluid. ${ }^{47,48}$

All of these results are very intriguing since they indicate that fundamentally new theories may not be needed to describe the physics of the nanoscale. At the same time the results are also quite puzzling, given the fact that bulk and nanoconfined liquids have very different phenomenology. In this connection, it is natural to wonder whether the similarities extend to a more fundamental or "microscopic" level. The difference between bulk and nanoconfined liquids is the external potential constituting the spatial confinement. The study of confinement is nevertheless complicated by the fact that the confining boundaries (the "walls") not only impose a geometrical constraint on the liquid, but also energetic costs. Several investigations have tried to separate the entropic and energetic contributions to the physics by studying, e.g., hardsphere walls, ${ }^{49}$ reflecting walls, ${ }^{50}$ amorphous walls, ${ }^{26,51}$ and more. With the above-mentioned similarities in mind, a "microscopic" conjecture motivated from these studies is that the interaction range for the wall-liquid and liquid-liquid interactions of the nanoconfined liquid is identical to that of the bulk liquid.

The relevant range of interaction for bulk liquids was considered long ago by traditional liquid-state theories, ${ }^{\mathbf{5 2 , 5 3}}$ and it is captured in the well-known Weeks-Chandler-Andersen (WCA) approximation ${ }^{53}$ in which the pair potential is truncated at the potential minimum. In this picture, the attractive pair forces of typical liquid-state configurations cancel to a good approximation and only contribute with a negative, virtually constant background potential. ${ }^{52}$ The relevance of a sharp 
distinction between the roles of the repulsive and attractive pair forces of the bulk liquid has, however, recently been questioned. $^{54-56}$ Instead, it has been suggested that the relevant distinction is between the forces within the first coordination shell (FCS) of molecules and those outside the FCS. ${ }^{55}$

The traditional bulk liquid-state picture has, in fact, been questioned for nonuniform liquids $;^{57}$ in particular, near the confining walls and close to drying transitions. Several promising theories, ${ }^{57-61}$ such as the Local Molecular Field (LMF) theory, ${ }^{57-60}$ have emerged to take into account the importance of the attractive forces in nonuniform liquids. We study here whether the FCS picture (that interactions beyond the FCS can safely be ignored ${ }^{55}$ ) of the bulk liquid state holds also for nanoconfined liquids, in particular for the wallliquid interactions. If this turns out to be the case for all liquids or, possibly, for a well-defined class of liquids, a more fundamental or microscopic similarity between bulk and nanoconfined liquids is established.

\section{Methods}

We applied GPU-optimized NVT molecular dynamics computer simulations ${ }^{62,63}$ (http://rumd.org) to the study of several atomic and molecular model systems in nanoconfinement. More specifically, we studied the single-component Lennard-Jones liquid (SCLJ), the Kob-Andersen binary LJ mixture ${ }^{64}$ (KABLJ), an asymmetric dumbbell model, ${ }^{65}$ and the Dzugutov liquid ${ }^{66}$ (see References for model details). The confinement itself is modeled as a symmetric slit-pore using the so-called Steele potential, ${ }^{36,67}$

$$
v_{9,3}(z)=\frac{4 \pi \varepsilon_{\mathrm{iw}} \rho_{\mathrm{w}} \sigma_{\mathrm{iw}}}{3}\left[\frac{1}{15}\left(\frac{\sigma_{\mathrm{iw}}}{z}\right)^{9}-\frac{1}{2}\left(\frac{\sigma_{\mathrm{iw}}}{z}\right)^{3}\right] .
$$

Here, $z$ is the distance between the divergence of the potential and the particle in question. $\sigma_{\mathrm{iw}}$ and $\varepsilon_{\mathrm{iw}}$ are parameters similar to those of the LJ potential, and $\rho_{\mathrm{w}}$ defines the density of the confining solid. The distance between the two walls of the slitpore is henceforth denoted $H$. Eqn (1) appears after considering the total interaction of a (liquid) LJ particle with a semi-infinite solid continuum of $\mathrm{LJ}$ particles ${ }^{67}$ and was used in early studies of confinement. ${ }^{68,69}$

Quantities are here and henceforth reported in dimensionless $\mathrm{LJ}$ units by setting $\sigma_{\mathrm{AA}}=1, \varepsilon_{\mathrm{AA}}=1$, etc. We use wallparameters $\sigma_{\mathrm{Aw}}=1, \varepsilon_{\mathrm{Aw}}=1$; in the case of binary systems $\sigma_{\mathrm{Bw}}=$ $\left(1+\sigma_{\mathrm{BB}}\right) / 2, \varepsilon_{\mathrm{Bw}}=\sqrt{1 \times \varepsilon_{\mathrm{BB}}}$. The density of the confining solid $\rho_{\mathrm{w}}$ is (in most cases) chosen to be equal to the total average slitpore density. ${ }^{36}$

In all simulations, we delimit the FCS cutoff of the liquidliquid (ll) and wall-liquid (wl) interactions from the distance of the first minimum of the contact-layer (i.e., the layer closest to the walls) radial distribution function (RDF) and density profile, respectively. This choice is analogous to that used for bulk liquids. ${ }^{55,70}$ To obtain reliable simulation results with FCS cutoffs, it was recently shown ${ }^{71}$ that it is necessary to ensure also continuity of forces at the cutoff. One method of achieving a continuous force is to use the so-called shifted-force (SF) cutoff

$$
f_{\mathrm{SF}}(r)= \begin{cases}f(r)-f\left(r_{\mathrm{c}}\right) & \text { if } r<r_{\mathrm{c}} \\ 0 & \text { if } r>r_{\mathrm{c}}\end{cases}
$$

which corresponds to adding the linear term $-v\left(r_{\mathrm{c}}\right)\left(r-r_{\mathrm{c}}\right)-$ $v\left(r_{\mathrm{c}}\right)$ to the original potential $v(r)$. The SF cutoff generally implies a significant change of the force, also below the cutoff. However, in the uniform bulk liquid the linear term to a good approximation sums to a constant over the nearest neighbors. ${ }^{72}$ The linear term of the SF cutoff thus does not affect the structure or dynamics of the simulation..$^{55,72}$

For nonuniform liquids it is not possible to apply a SF cutoff to the liquid alone as the molecules near the walls are missing "half" a coordination shell. In other words, the linear term of the SF approach does not sum to a constant for the nearest neighbors of a particle in the vicinity of a wall. We have found empirically, however, that applying a SF cutoff also to the wall-liquid interactions of eqn (1) in combination with adding an additional force term achieves a nearconstant linear term. The additional force term added is given by

$$
\Delta f_{\mathrm{FCS}}=\left|f_{\mathrm{PAIR}}\left(r_{\mathrm{ll}, \mathrm{FCS}}\right)-f_{\mathrm{WALL}}\left(r_{\mathrm{wl}, \mathrm{FCS}}\right)\right|,
$$

in which $r_{1 \mathrm{l}, \mathrm{FCS}}$ and $r_{\mathrm{wl}, \mathrm{FCS}}$ are, respectively, the distance of the FCS (see previously) for the liquid-liquid and wall-liquid interactions (and are thus not free parameters). The quantity $f_{\text {WALL }}$ is given by eqn (1) and $f_{\text {PAIR }}$ depends on the particular model studied. To summarize, the FCS forces in simulations are given by

$$
f_{\mathrm{PAIR}, \mathrm{FCS}}(r)= \begin{cases}f_{\mathrm{PAIR}}(r)-f_{\mathrm{PAIR}}\left(r_{1 \mathrm{ll}, \mathrm{FCS}}\right) & \text { if } r<r_{1 \mathrm{ll}, \mathrm{FCS}}, \\ 0 & \text { if } r>r_{1 \mathrm{ll}, \mathrm{FCS}},\end{cases}
$$

and

$f_{\mathrm{WALL}, \mathrm{FCS}}(r)= \begin{cases}f_{\mathrm{WALL}}(r)-f_{\mathrm{WALL}}\left(r_{\mathrm{wl}, \mathrm{FCS}}\right)-\Delta f_{\mathrm{FCS}} & \text { if } r<r_{\mathrm{wl}, \mathrm{FCS}} \\ 0 & \text { if } r>r_{\mathrm{wl}, \mathrm{FCS}}\end{cases}$

We have no rigorous arguments for using eqn (3) and as shown above it leaves $f_{\mathrm{WALL}, \mathrm{FCS}}$ discontinuous at the cutoff. Nevertheless, the described cutoff method was applied to all FCS simulations.

\section{Results}

To set the stage of investigation we present in Fig. 1 the impact of the slit-pore confinement (magenta) on the bulk SCLJ liquid (blue). Fig. 1(a) shows the density profile and Fig. 1(b) shows the lateral RDF. The RDF in nanoconfinement is calculated for the contact layer, and the comparison is performed at the same average density and temperature. The slit-pore has a significant impact on the structure of the liquid leading to large density oscillations near the walls. The (lateral) RDF is also affected by the walls, showing a higher degree of ordering in the liquid. The latter confirms results from other computer simulations of nanoconfined liquids..$^{22,69}$ 

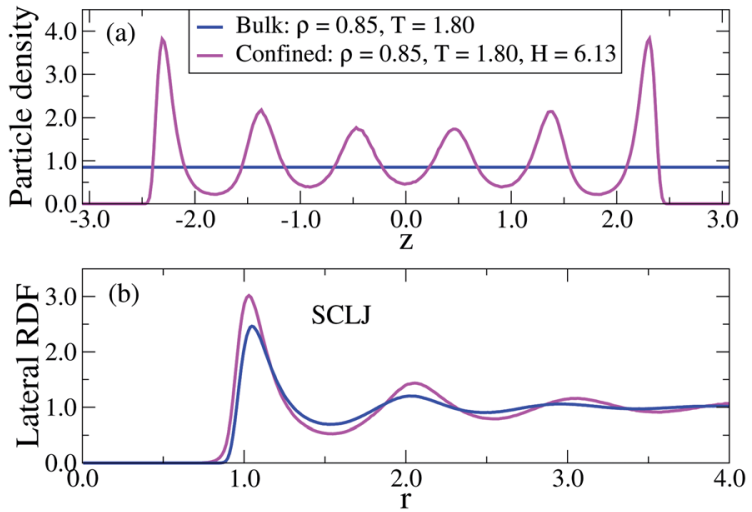

Fig. 1 Comparing slit-pore confinement (magenta) and bulk SCLJ liquid (blue) at $\rho=0.85$ and $T=1.80$. (a) Average density profiles. (b) Lateral RDFs. The RDF in nanoconfinement is calculated for the contact layer, i.e., the layer closest to the walls, showing a structure distinct from the bulk liquid.

From these observations it is clear that the microscopic physics near the walls is quite different from that of the bulk liquid, as expected due to the wall-liquid interactions.

\section{A. Simulations of FCS cutoffs}

We now proceed to test the bulk FCS picture in nanoconfinement, i.e., the hypothesis that interactions may be ignored beyond the first coordination shell. Fig. 2 shows density profiles and contact-layer RDFs and mean-square displacements (MSDs) for the SCLJ confined state point of Fig. 1. The black curves give a simulation with a large pair potential cutoff $\left(r_{\mathrm{c}}=5.00\right)$ and no cutoff for the wall-liquid interactions. The red dots give results for the FCS cutoff for both liquid-liquid and wall-liquid interactions. The FCS cutoff simulations capture the relevant physics for the entire slit-pore, even though the walls modify the structure of the liquid significantly compared to the bulk liquid (Fig. 1).

To further test the FCS picture for nanoconfined liquids, Fig. 3 shows similar computer simulations for the KABLJ mixture $^{64}$ at $\rho=1.20, T=1.40, H=5.97$. Fig. 3(a) shows A-particle density profiles, Fig. 3(b) shows lateral RDFs of the contact layer, and Fig. 3(c) shows lateral A-particle incoherent intermediate scattering functions (ISFs) of the contact layer. The physics near the walls is seen to be captured very well using the FCS cutoffs. It should be noted here that a defective crystal was obtained as the system was cooled from the melt; the ISF of the contact layer thus shows a two-step-like relaxation similar to that of a supercooled liquid.

Proceeding to study in Fig. 4 a small-molecule liquid (the asymmetric dumbbell model $^{65}$ ), we show in Fig. 4 lateral contact-layer RDFs and ISFs. Slight deviations are seen between the FCS cutoff (see Methods section) and the reference cutoff for the ISF; we assume these to be due to the approximative linearcancellation term added to the force from the walls (see eqn (3)).

One may wonder: how general are these results? An FCS cutoff of the bulk liquid is shown in ref. 55 to work well only when the liquid is what is now called "Roskilde simple"
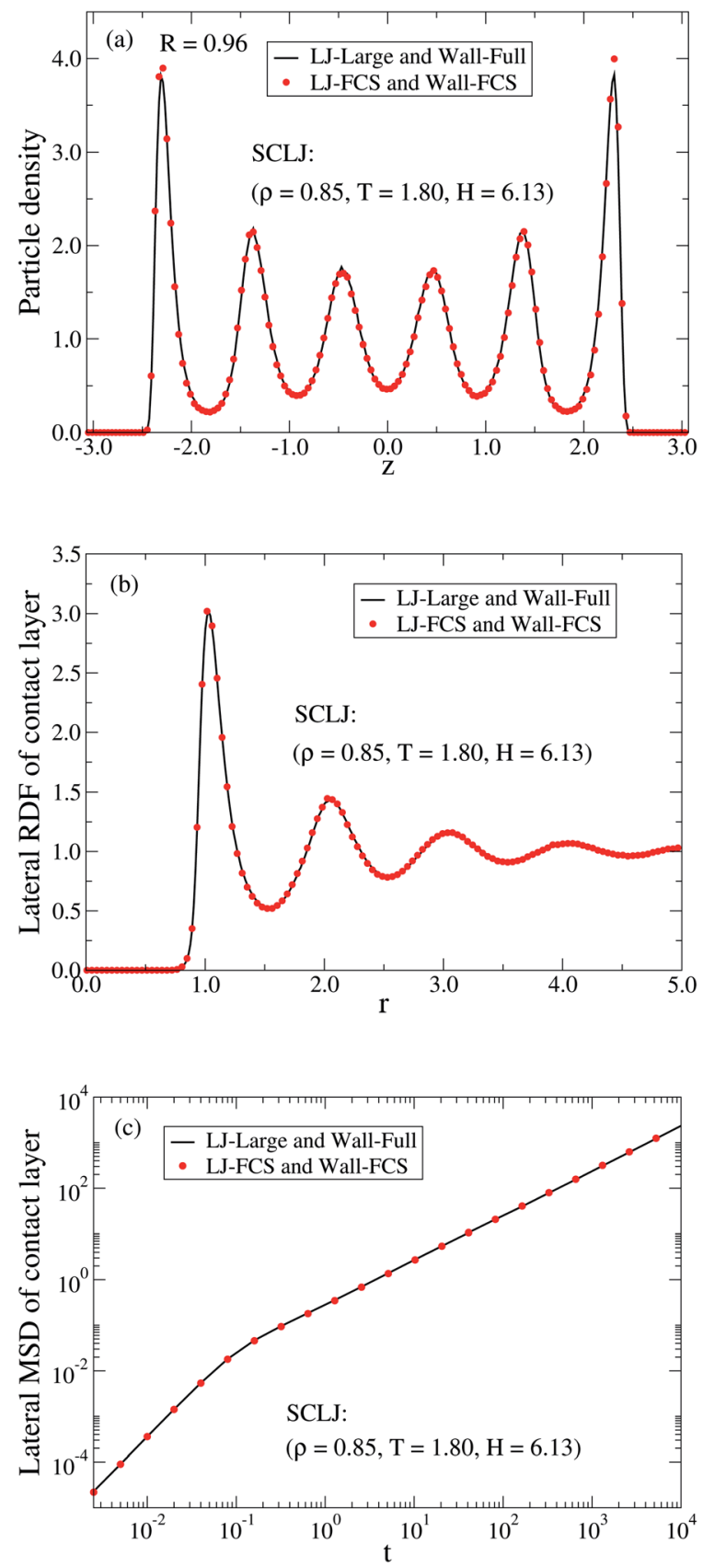

Fig. 2 FCS simulations for the SCLJ liquid in a slit-pore at $\rho=0.85, T=$ 1.80, $H=6.13$. The black curves give a simulation with a large pair potential cutoff $\left(r_{c}=5.00\right)$ in a combination with no cutoff for the wall-liquid interactions. The red dots give results for a cutoff at the FCS for the liquid-liquid and wall-liquid interactions $\left(r_{l, \mathrm{FCS}}=1.549\right.$ and $r_{\text {wl,FCS }}=1.250$ ). (a) Density profiles. $R$ is the virial potential-energy correlation coefficient, ${ }^{73}$ defined in eqn (6). (b) Lateral RDFs of the contact layer. (c) Lateral mean-square displacements (MSDs) of the contact layer.

(previously referred to as "strongly correlating", a name that however gave rise to confusion with strongly correlated quantum systems), defined ${ }^{73}$ by having the NVT virial/potential energy correlation coefficient (which depends on the state point), 

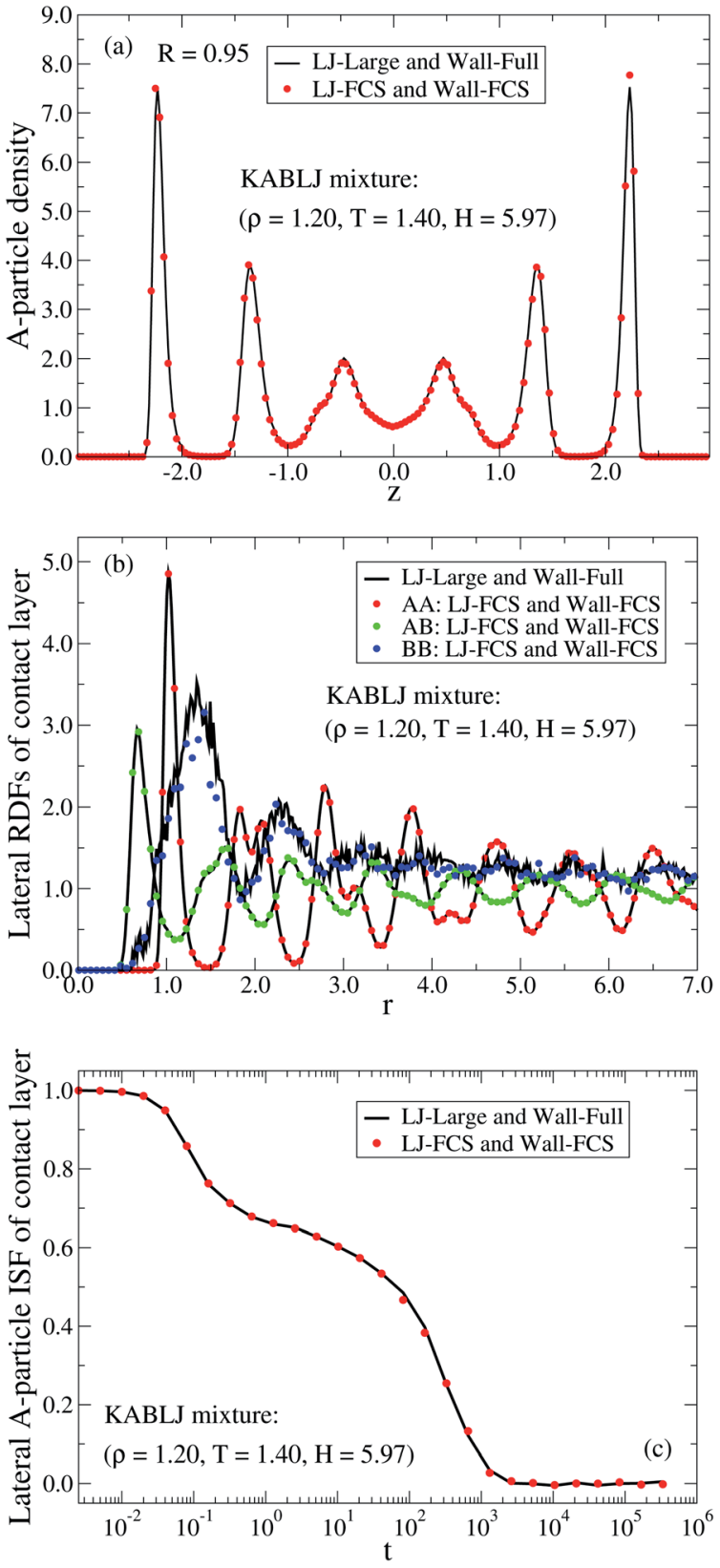

Fig. 3 FCS simulations for the KABLJ mixture in a slit-pore at $\rho=1.20$, $T=1.40, H=5.97\left(r_{\mathrm{C}}=8.00, r_{\mathrm{ll}, \mathrm{FCS}}=1.479\right.$, and $\left.r_{\mathrm{wl}, \mathrm{FCS}}=1.252\right)$. The virial potential-energy correlation coefficient $R$ is defined in eqn (6). (a) Density profiles. (b) Lateral RDFs of the contact layer. The BB-particle RDF has more noise than the other particle-particle RDFs. (c) Lateral A-particle ISFs of the contact layer.

$$
R=\frac{\langle\Delta W \Delta U\rangle}{\sqrt{\left\langle(\Delta W)^{2}\right\rangle} \sqrt{\left\langle(\Delta U)^{2}\right\rangle}},
$$

above 0.90 . Only inverse-power-law fluids are perfectly correlating $(R=1)$, but many models, ${ }^{55,73}$ e.g., the SCLJ liquid, KABLJ mixture, Lewis-Wahström OTP, ${ }^{74}$ as well as some experimental liquids ${ }^{75,76}$ have been shown to be Roskilde-simple. Roskilde liquids are believed to include most or all van der Waals and metallic liquids as well as weakly ionic or dipolar liquids,
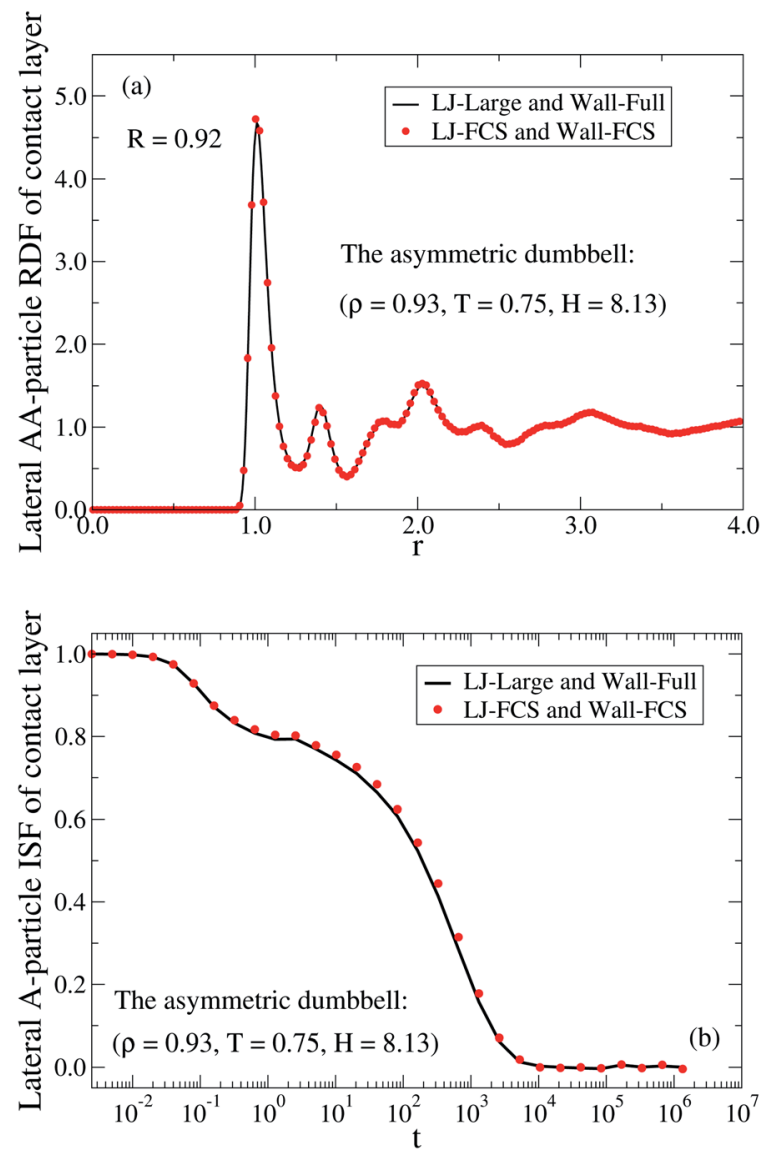

Fig. 4 FCS simulations for the asymmetric dumbbell model in a slitpore at $\rho=0.93, T=0.75, H=8.13\left(r_{\mathrm{C}}=4.00, r_{\mathrm{ll}, \mathrm{FCS}}=1.565\right.$, and $r_{\mathrm{wl}, \mathrm{FCS}}$ $=1.015)$. $R$ is defined in eqn (6). (a) Lateral RDFs of the contact layer. (b) Lateral ISFs of the contact layer.

whereas covalently, hydrogen-bonding or strongly ionic or dipolar liquids are generally not Roskilde simple. ${ }^{73}$ The latter reflects the fact that directional interactions destroy the strong virial/potential energy correlation.

In all the examples studied so far in the present paper the liquid exhibits a strong virial/potential energy correlation in both bulk and confinement ( $R \geq 0.90$; see figures). It is possible that as the virial/potential energy correlation decreases, the FCS cutoff of the nanoconfined liquid becomes a worse approximation. To test this conjecture, we proceed to study non-Roskilde liquids. One possible way of obtaining such a system is to decrease the density of the SCLJ liquid (recall that $R$ depends on the state point). Doing so, we obtain the results shown in Fig. 5; the correlation coefficient is here $R=0.74$. We now observe larger deviations with the FCS cutoff, which is consistent with the above conjecture.

As a further example, we show in Fig. 6 simulation results for the Dzugutov liquid ${ }^{66}$ which is also a non-Roskilde liquid with $R$ $=0.70$. Here, the failure of the FCS cutoff in nanoconfinement is dramatic.

\section{B. Simulations of WCA cutoffs}

Having established that for Roskilde liquids the interactions beyond the FCS may be ignored for both the liquid-liquid and 


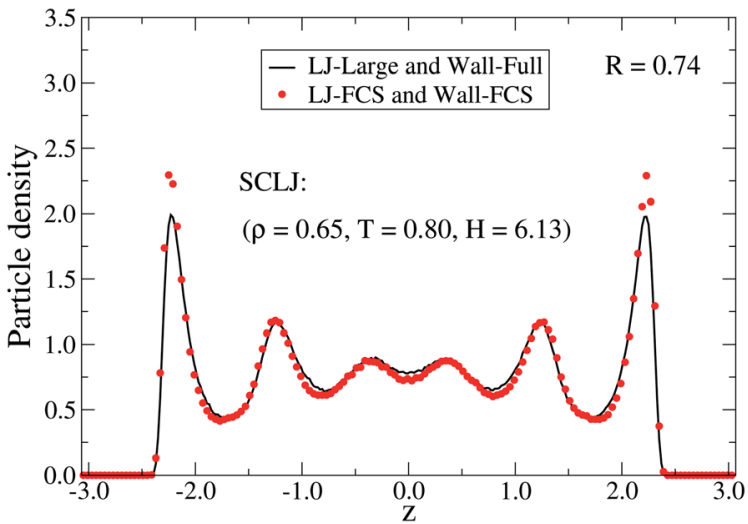

Fig. 5 FCS simulations for the SCLJ liquid in a slit-pore at $\rho=0.65, T=$ $0.80, H=6.13\left(r_{\mathrm{C}}=7.50, r_{\mathrm{LLCS}}=1.660\right.$, and $\left.r_{\mathrm{wL}, \mathrm{FCS}}=1.377\right) . R$ is defined in eqn (6). At this state point, the SCLJ liquid is not Roskilde simple (because $R<0.90$ ), and the FCS cutoff does not work well.

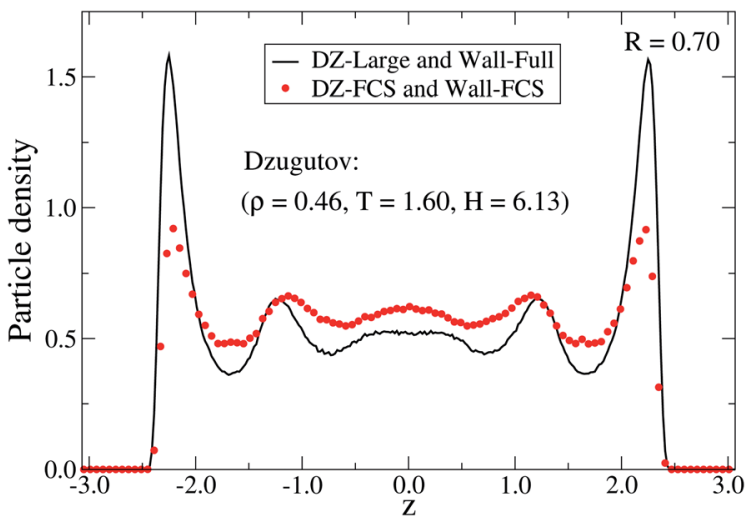

Fig. 6 FCS simulations for the Dzugutov liquid in a slit-pore at $\rho=$ $0.46, T=1.60, H=6.13\left(r_{\mathrm{C}}=1.94, r_{\mathrm{ll}, \mathrm{FCS}}=1.616\right.$, and $\left.r_{\mathrm{wl}, \mathrm{FCS}}=1.406\right) . R$ is defined in eqn (6). The Dzugutov liquid is not a Roskilde liquid.

wall-liquid interactions, we now consider the effect of decreasing the cutoff below the FCS radius. According to the WCA philosophy $^{53}$ it should be possible to cut the potentials at the potential minima and still obtain the correct physics. Applying this method to simulations of confined liquids, we obtain the results of Fig. 7 for the KABLJ mixture. We now observe significant discrepancy with the reference simulation, confirming the results of previous simulations of confined liquids..$^{57,58}$

The discrepancy is largest near the walls. To understand this behavior in more detail, we show in Fig. 8 results obtained by applying the WCA approximation only to the wall-liquid interaction. Interestingly, here no discrepancy is noted.

From these results one might be tempted to conclude that the FCS is not the fundamental distance for the wall-liquid interactions. The FCS does, however, depend on the density and it is thus possible to perform simulations in which the FCS cutoff is below the potential minimum.

Fig. 9 presents results for the confined SCLJ liquid at a highdensity state point. The potential minima are, respectively, for the liquid-liquid and wall-liquid interactions at $2^{1 / 6} \sigma \approx 1.12$
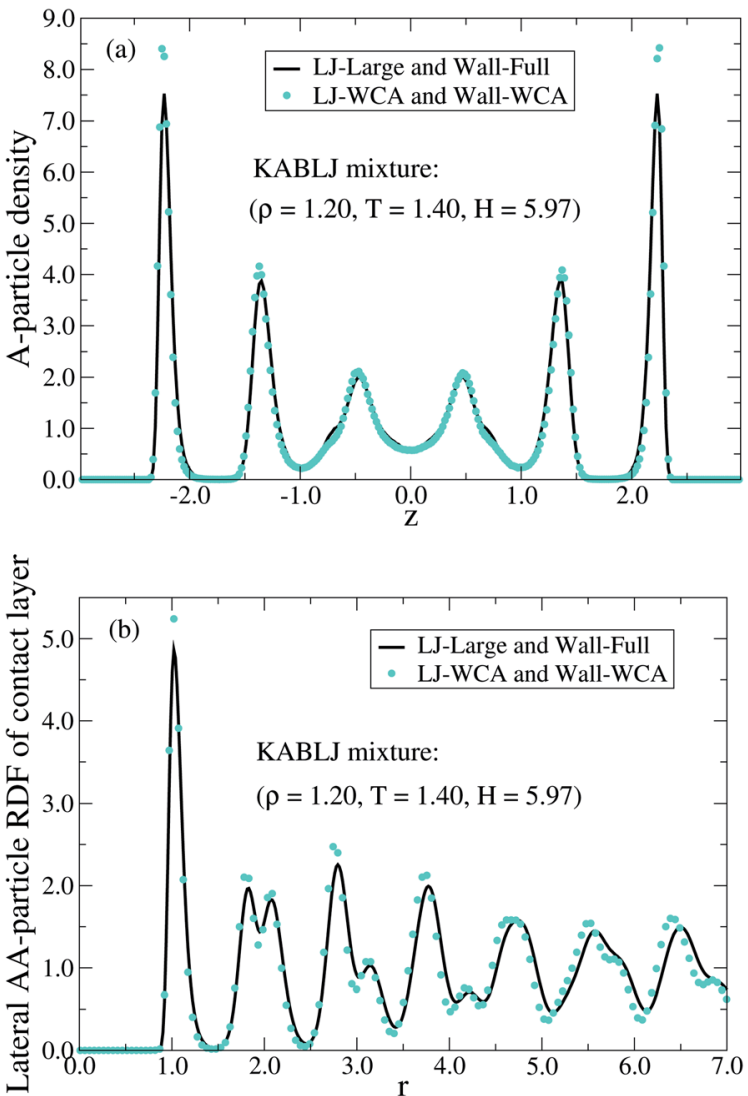

Fig. 7 Simulations of the KABLJ mixture confined to a slit-pore at $\rho=$ $1.20, T=1.40, H=5.97$ for which the WCA-cutoff method is applied to both the liquid-liquid and wall-liquid interactions. (a) A-particle density profiles. (b) Lateral AA-particle RDFs of the contact layer.

and $(2 / 5)^{1 / 6} \sigma_{\mathrm{w}} \approx 0.86$. The FCS cutoffs are, respectively, $r_{\mathrm{ll}, \mathrm{FCS}}=$ 0.934 and $r_{\mathrm{wl}, \mathrm{FCS}}=0.669$ and thus smaller than the location of the potential minima. A perfect agreement with the reference simulation is obtained, confirming that the FCS indeed defines the fundamental cutoff distance, also for the wall-liquid interactions.

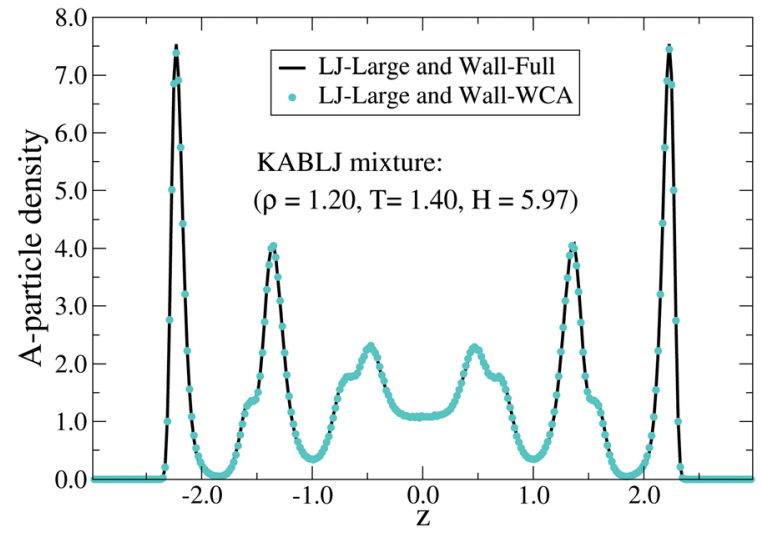

Fig. 8 Simulations of the KABLJ mixture confined to a slit-pore at $\rho=$ 1.20, $T=1.40, H=5.97$. The WCA-cutoff method is here applied only to the wall-liquid interactions. 


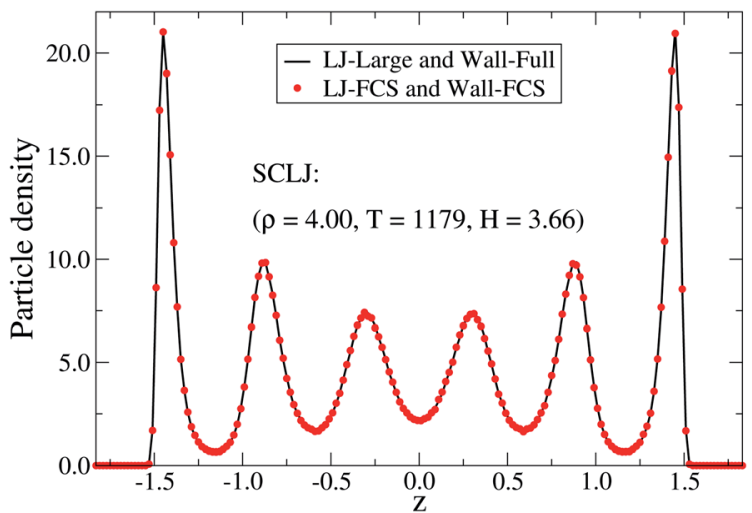

Fig. 9 FCS simulations for the SCLJ liquid in a slit-pore at $\rho=4.00, T=$ $1179, H=3.66$. At this state point the FCSs are pushed to very small distances $\left(r_{\mathrm{ll}, \mathrm{FCS}}=0.934\right.$ and $\left.r_{\mathrm{Wl}, \mathrm{FCS}}=0.669\right)$, well below the location of the potential minima $\left(2^{1 / 6} \sigma \approx 1.12\right.$ and $\left.(2 / 5)^{1 / 6} \sigma_{\mathrm{w}} \approx 0.86\right)$.

\section{Conclusions}

The results presented here suggest that bulk and nanoconfined liquids are more similar than traditionally believed. The physics as exemplified from the range of interactions is as in the bulk liquid as long as the liquid is Roskilde simple in both bulk and nanoconfinement. In general, covalently, hydrogen-bonding or strongly ionic or dipolar liquids are not in this class. For these liquids we do not expect a similar strong connection between bulk and nanoconfined systems. This conjecture is consistent with the fact that second- and higher coordination shell effects play an important role in such liquids. ${ }^{77-79}$

The current study focused on the structure and dynamics of the simulated systems. A property often used to characterize the "structure" of nonuniform liquids is the solvation force ${ }^{69}$ (normal pressure). This quantity (and others) is different in the FCS system due to the truncation. It should be noted that some thermodynamic quantities are unchanged in the FCS system. As an example, the excess entropy and excess isochoric heat capacity are, to a good approximation, unchanged.

Recently, Watanabe et $a .^{22}$ showed that the dynamics of a confined fluid system (which in the bulk is a Roskilde liquid) as a function of the distance to the walls can be described to a good approximation using the magnitude of the medium-range crystalline order ${ }^{80}$ (MRCO). In this approach, the relevant range of interaction for the wall-liquid interactions is given by the correlation length of MRCO, itself, just as in the bulk liquid. The MRCO perspective is thus fully consistent with the FCS picture proposed here.

In the present study we modeled the wall via a potential derived from a semi-infinite solid continuum of $\mathrm{LJ}$ particles. The SCLJ solid is a Roskilde system. ${ }^{72,73}$ Additional studies of more diverse wall potentials are needed to fully clarify the FCS conjecture proposed here. It is, in our opinion, likely that a requirement of analyticity must be placed on the external potential - similar to the bulk liquid. ${ }^{55}$ This is, however, not a crucial restriction, since nature is expected to be analytic. In addition, we applied an approximative linear-cancellation term near the walls (eqn (3)), which works very well for many systems. We have encountered state points for the KABLJ mixture where the cancellation was not perfect (e.g., $\rho=1.14$ and $T=1.40$; data not shown). More work is needed to clarify this issue.

The FCS approach to bulk and nonuniform liquids as detailed in this and prior papers ${ }^{54,55}$ provides fundamental insights into the physics of Roskilde liquids. The FCS approach, however, suffers certain limitations as a practical tool; specifically, one would often like to have potentials that do not depend on the state point. This is, however, the price paid in unraveling the underlying physics. It is likely that other theories, such as DFT, may be used to predict the location of the FCS prior to simulation.

In experiments it is often observed that even simple liquids confined to the nanoscale can exhibit unusually rich behavior. ${ }^{\mathbf{8 1 - 8 3}}$ In the viewpoint presented here this fact may be due to the confining walls interfering with the property of being a Roskilde liquid (e.g., a van der Waals bonded liquid). It would thus in experiments be interesting to investigate systematic trends by varying the confinement of known Roskilde liquids..$^{55,75}$ In this connection, we also welcome additional simulation studies of a wide spectrum of Roskilde liquids/ external potentials to further test the FCS conjecture proposed here. Finally, we note that more theoretical work is needed to explain why Roskilde liquids obey the FCS conjecture.

\section{Acknowledgements}

The center for viscous liquid dynamics "Glass and Time" is sponsored by the Danish National Research Foundation via Grant no. DNRF61. We are grateful to John D. Weeks and Richard Remsing for stimulating correspondence concerning the role of attractive forces in nonuniform liquids.

\section{References}

1 B. Bhushan, J. N. Israelachvili and U. Landman, Nature, 1995, 374, 607.

2 G. M. Whitesides, Nature, 2006, 442, 368.

3 J. M. Drake and J. Klafter, Phys. Today, 1990, 43, 46.

4 S. Granick, Science, 1991, 253, 1374.

5 D. Morineau, Y. Xia and C. Alba-Simionesco, J. Chem. Phys., 2002, 117, 8966.

6 C. L. Jackson and G. B. McKenna, J. Non-Cryst. Solids, 1991, 131-133, 221.

7 V. Teboul and C. Alba-Simionesco, Chem. Phys., 2005, 317, 245.

8 M. Alcoutlabi and G. B. McKenna, J. Phys.: Condens. Matter, 2005, 17, R461.

9 B. Coasne, C. Alba-Simionesco, F. Audonnet, G. Dosseh and K. E. Gubbins, Phys. Chem. Chem. Phys., 2011, 13, 3748.

10 R. Richert, Annu. Rev. Phys. Chem., 2011, 62, 65.

11 J. L. Keddie, R. A. L. Jones and R. A. Cory, Europhys. Lett., 1994, 27, 59.

12 A. Serghei, M. Tress and F. Kremer, Macromolecules, 2006, 39, 9385. 
13 J. A. Forrest and K. Dalnoki-Veress, Adv. Colloid Interface Sci., 2001, 94, 167.

14 C. J. Ellison and J. M. Torkelson, Nat. Mater., 2003, 2, 695.

15 P. Rittigstein, R. D. Priestley, L. J. Broadbelt and J. M. Torkelson, Nat. Mater., 2007, 6, 278.

16 K. Paeng, R. Richert and M. D. Ediger, Soft Matter, 2012, 8, 819.

17 C. Iacob, J. R. Sangoro, W. K. Kipnusu, R. Valiullin, J. Kärger and F. Kremer, Soft Matter, 2012, 8, 289.

18 Q. Ji, R. Lefort, A. Ghoufi and D. Morineau, Chem. Phys. Lett., 2009, 482, 234.

19 C. R. Nugent, K. V. Edmond, H. N. Patel and E. R. Weeks, Phys. Rev. Lett., 2007, 99, 025702.

20 H. B. Eral, D. van den Ende, F. Mugele and M. H. G. Duits, Phys. Rev. E: Stat., Nonlinear, Soft Matter Phys., 2009, 80, 061403.

21 V. N. Michailidou, G. Petekidis, J. W. Swan and J. F. Brady, Phys. Rev. Lett., 2009, 102, 068302.

22 K. Watanabe, T. Kawasaki and H. Tanaka, Nat. Mater., 2011, 10, 512.

23 K. V. Edmond, C. R. Nugent and E. R. Weeks, Phys. Rev. E: Stat., Nonlinear, Soft Matter Phys., 2012, 85, 041401.

24 T. Fehr and H. Löwen, Phys. Rev. E: Stat. Phys., Plasmas, Fluids, Relat. Interdiscip. Top., 1995, 52, 4016.

25 J. A. Torres, P. F. Nealey and J. J. de Pablo, Phys. Rev. Lett., 2000, 85, 3221.

26 P. Scheidler, W. Kob and K. Binder, J. Phys. IV, 2000, 10, 33.

27 F. W. Starr, T. B. Schrøder and S. C. Glotzer, Macromolecules, 2002, 35, 4481.

28 A. R. C. Baljon, M. H. M. V. Weert, R. B. DeGraaff and R. Khare, Macromolecules, 2005, 38, 2391.

29 J. Kurzidim, D. Coslovich and G. Kahl, Phys. Rev. Lett., 2009, 103, 138303.

30 F. W. Starr and J. F. Douglas, Phys. Rev. Lett., 2011, 106, 115702.

31 B. A. P. Betancourt, J. F. Douglas and F. W. Starr, Soft Matter, 2013, 9, 241.

32 Y. Rosenfeld, Phys. Rev. A, 1977, 15, 2545.

33 J. Mittal, J. R. Errington and T. M. Truskett, Phys. Rev. Lett., 2006, 96, 177804 .

34 R. Chopra, T. M. Truskett and J. R. Errington, Phys. Rev. E: Stat., Nonlinear, Soft Matter Phys., 2010, 82, 041201.

35 G. Goel, W. P. Krekelberg, M. J. Pond, J. Mittal, V. K. Shen, J. R. Errington and T. M. Truskett, J. Stat. Mech.: Theory Exp., 2009, 04006.

36 T. S. Ingebrigtsen, J. R. Errington, T. M. Truskett and J. C. Dyre, Phys. Rev. Lett., 2013, 111, 235901.

37 Y. Rosenfeld and P. Tarazona, Mol. Phys., 1998, 95, 141.

38 T. S. Ingebrigtsen, A. A. Veldhorst, T. B. Schrøder and J. C. Dyre, J. Chem. Phys., 2013, 139, 171101.

39 W. E. Alley and B. J. Alder, Phys. Rev. A, 1983, 27, 3158.

40 K. P. Travis, B. D. Todd and D. J. Evans, Phys. Rev. E: Stat. Phys., Plasmas, Fluids, Relat. Interdiscip. Top., 1997, 55, 4288.

41 J. S. Hansen, P. J. Daivis, K. P. Travis and B. D. Todd, Phys. Rev. E: Stat., Nonlinear, Soft Matter Phys., 2007, 76, 041121.
42 J. S. Hansen, P. J. Daivis, J. C. Dyre, B. D. Todd and H. Bruus, J. Chem. Phys., 2013, 138, 034503.

43 W. Götze, Complex Dynamics of Glass-Forming Liquids: A Mode-Coupling theory, Oxford University Press, New York, 1st edn, 2009.

44 V. Krakoviack, Phys. Rev. Lett., 2005, 94, 065703.

45 G. Biroli, J.-P. Bouchaud, K. Miyazaki and D. R. Reichman, Phys. Rev. Lett., 2006, 97, 195701.

46 S. Lang, R. Schilling, V. Krakoviack and T. Franosch, Phys. Rev. E: Stat., Nonlinear, Soft Matter Phys., 2012, 86, 021502.

47 R. Evans, Adv. Phys., 1979, 28, 143.

48 J. Mittal, J. R. Errington and T. M. Truskett, J. Phys. Chem. B, 2007, 111, 10054.

49 D. J. Diestler, M. Schoen, A. W. Hertzner and J. H. Cushman, J. Chem. Phys., 1991, 95, 5432.

50 W. P. Krekelberg, V. K. Shen, J. R. Errington and T. M. Truskett, J. Chem. Phys., 2011, 135, 154502.

51 P. Scheidler, W. Kob and K. Binder, Europhys. Lett., 2000, 52, 277.

52 B. Widom, Science, 1967, 157, 3787.

53 J. D. Weeks, D. Chandler and H. C. Andersen, J. Chem. Phys., 1971, 54, 5237.

54 S. Toxvaerd and J. C. Dyre, J. Chem. Phys., 2011, 135, 134501.

55 T. S. Ingebrigtsen, T. B. Schrøder and J. C. Dyre, Phys. Rev. X, 2012, 2, 011011.

56 L. Bøhling, A. A. Veldhorst, T. S. Ingebrigtsen, N. P. Bailey, J. S. Hansen, S. Toxvaerd, T. B. Schrøder and J. C. Dyre, J. Phys.: Condens. Matter, 2013, 25, 032101.

57 J. D. Weeks, R. L. B. Selinger and J. Q. Broughton, Phys. Rev. Lett., 1995, 75, 2694.

58 J. D. Weeks, K. Vollmayr and K. Katsov, Phys. A, 1997, 244, 461.

59 J. D. Weeks, K. Katsov and K. Vollmayr, Phys. Rev. Lett., 1998, 81, 4400.

60 J. M. Rodgers and J. D. Weeks, J. Phys.: Condens. Matter, 2008, 20, 494206.

61 H. Wang, C. Schütte and P. Zhang, Phys. Rev. E: Stat., Nonlinear, Soft Matter Phys., 2012, 86, 026704.

62 S. Toxvaerd, Mol. Phys., 1991, 72, 159.

63 S. Toxvaerd, O. J. Heilmann, T. Ingebrigtsen, T. B. Schrøder and J. C. Dyre, J. Chem. Phys., 2009, 131, 064102.

64 W. Kob and H. C. Andersen, Phys. Rev. E: Stat. Phys., Plasmas, Fluids, Relat. Interdiscip. Top., 1995, 51, 4626.

65 T. B. Schrøder, U. R. Pedersen, N. P. Bailey, S. Toxvaerd and J. C. Dyre, Phys. Rev. E: Stat., Nonlinear, Soft Matter Phys., 2009, 80, 041502.

66 M. Dzugutov, Phys. Rev. A, 1992, 46, R2984.

67 W. A. Steele, Surf. Sci., 1973, 36, 317.

68 S. Toxvaerd, J. Chem. Phys., 1981, 74, 1998.

69 M. Schoen, D. J. Diestler and J. H. Cushman, J. Chem. Phys., 1987, 87, 5464.

70 D. Chandler, Introduction to Modern Statistical Mechanics, Oxford University Press, New York, 1987.

71 S. Toxvaerd and J. C. Dyre, J. Chem. Phys., 2011, 134, 081102.

72 N. P. Bailey, U. R. Pedersen, N. Gnan, T. B. Schrøder and J. C. Dyre, J. Chem. Phys., 2008, 129, 184508. 
73 N. P. Bailey, U. R. Pedersen, N. Gnan, T. B. Schrøder and J. C. Dyre, J. Chem. Phys., 2008, 129, 184507.

74 L. J. Lewis and G. Wahnström, J. Non-Cryst. Solids, 1994, 172174, 69.

75 D. Gundermann, U. R. Pedersen, T. Hecksher, N. P. Bailey, B. Jakobsen, T. Christensen, N. B. Olsen, T. B. Schrøder, D. Fragiadakis, R. Casalini, C. M. Roland, J. C. Dyre and K. Niss, Nat. Phys., 2011, 7, 816.

76 L. A. Roed, D. Gundermann, J. C. Dyre and K. Niss, J. Chem. Phys., 2013, 139, 101101.

77 Z. Yan, S. V. Buldyrev, P. Kumar, N. Giovambattista, P. G. Debenedetti and H. E. Stanley, Phys. Rev. E: Stat., Nonlinear, Soft Matter Phys., 2007, 76, 051201.
78 W. P. Krekelberg, J. Mittal, V. Ganesan and T. M. Truskett, J. Chem. Phys., 2007, 127, 044502.

79 W. P. Krekelberg, J. Mittal, V. Ganesan and T. M. Truskett, Phys. Rev. E: Stat., Nonlinear, Soft Matter Phys., 2008, 77, 041201.

80 H. Shintani and H. Tanaka, Nat. Phys., 2006, 2, 200.

81 V. Teboul and C. A. Simionesco, J. Phys.: Condens. Matter, 2002, 14, 5699.

82 G. Dosseh, C. L. Quellec, N. Brodie-linder, C. Albasimionesco, W. Haeussler and P. Levitz, J. Non-Cryst. Solids, 2006, 352, 4964.

83 C. L. Quellec, G. Dosseh, F. Audonnet, N. Brodie-Linder, C. Alba-Simionesco, W. Häussler and B. Frick, Eur. Phys. J.: Spec. Top., 2007, 141, 11. 